\title{
Chinese college students' beliefs about instructor and peer feedback functions
}

\author{
Ying Zhan \\ The Education University of Hong Kong
}

\begin{abstract}
Feedback has been increasingly conceptualized as a dialogical process where students interpret the provided information through interaction with comment providers and use it to enhance their learning. A major challenge for the development of sustainable feedback is closely related to how students think about it. Discrepancies in the understanding of feedback between academics and students might exist. If students do not acknowledge feedback as a dialogic process as academics do, the feedback produced will be in vain. This study explored how 25 Chinese university students made sense of instructor and peer feedback following their English group presentations. Retrospective journals and post-journal interviews were used to collect the data in this study. Thematic analysis was employed to analyse journal entries and transcribed interview data.
\end{abstract}

According to data analysis, the participants perceived more judging and encouraging functions than improving functions. It seems that Chinese learners are more likely to attach affective functions to feedback than Western learners. Variation also existed in the perceived functions of instructor and peer feedback. Overall, the participants tended to regard feedback in a conventional rather than a sustainable way.

Imbalanced power-relations, face and group harmony appeared to inhibit the interactions between instructors and students as well as among peers, which in turn made them see the feedback as being of limited use in moving their learning forward. This shows that cultural values in Confucianism may pose great challenges for the development of sustainable feedback in the Chinese context. Instructors' feedback practice and students' past learning and examination experiences seemed to prevent the participants from acting upon the feedback, which in turn they obstructed the improving function of feedback. The participants' pragmatic learning dispositions and emphasis on effort in their learning also reflects the influence of Confucianism on their views on feedback functions.

This study sheds light on college students' complex thinking about feedback in a nonAnglophone context which has been neglected in the feedback literature and has implications for educators and researchers in facilitating sustainable feedback in the Chinese context and the non-Chinese contexts where Chinese students study. 